\title{
A differential-outcome effect in pigeons using spatial hedonically nondifferential outcomes
}

\author{
Andrea M. Friedrich • Thomas R. Zentall
}

Published online: 29 January 2011

(C) Psychonomic Society, Inc. 2011

\begin{abstract}
We examined the extent to which nonhedonically different differential outcomes involving feeder location control pigeons' comparison choices in matching to sample. In Experiment 1, we showed that differential feeder location outcomes associated with each of two samples can facilitate delayed-matching accuracy. In Experiment 2, we found positive transfer following training on two matching tasks with differential feeder location outcomes when samples from one task were replaced by samples from the other task. In Experiment 3, we found that when differential-outcome expectations could no longer serve as the cues for comparison choice, sample stimuli continued to exert some control over choice of comparisons. The results indicate that differential outcomes (involving feeder location) that presumably do not differ in hedonic value are sufficient to control comparison choice. Thus, the differential hedonic value of the outcome elicited by the sample does not appear to be a requirement of the differential-outcome effect. Furthermore, these differential outcomes appear to augment matching accuracy, but they do not eliminate control by the samples.
\end{abstract}

Keywords Differential outcomes $\cdot$ Hedonically nondifferential $\cdot$ Matching-to-sample $\cdot$ Delayed matching . Feeder location $\cdot$ Transfer of training $\cdot$ Pigeons

Traditionally, learning theorists have assumed that the role of reinforcement is to strengthen, or "stamp in," the association between a stimulus and the response that follows. It is now

\footnotetext{
A. M. Friedrich • T. R. Zentall $(\bowtie)$

Department of Psychology, University of Kentucky,

Lexington, KY 40506-0044, USA

e-mail: zentall@uky.edu
}

clear, however, that the association between the response and outcome that follows plays an important role in learning. In fact, it is now widely accepted that outcome anticipation can serve as the basis of response selection (e.g., Peterson, 1984). In a seminal study, Trapold (1970) trained rats to press a right lever after hearing a tone and to press a left lever after hearing a click. For half of the subjects, correct right-lever choices were reinforced with one type of reinforcer (e.g., pellets), while correct left-lever choices were reinforced with another type of reinforcer (e.g., sucrose). For the remaining subjects, both right- and left-lever choices were reinforced with the same type of reinforcer (either all pellets or all sucrose).

Trapold (1970) found that rats for which the two responses were differentially reinforced with pellets and sucrose acquired the task more quickly and performed it more accurately than rats for which the two responses were reinforced with the same type of reinforcer. This finding suggests that animals may use the reinforcer, which occurs only after the response, to help them choose which response to perform. In other words, they can use the anticipation of an outcome associated with an initial stimulus as a discriminative stimulus. This facilitation in acquisition observed when responses are differentially reinforced became known as the differential-outcome effect.

It has also been observed that the use of differential outcomes can improve delayed-matching accuracy. That is, either the presumed outcome expectation appears to facilitate memory for the sample, or the expected outcome itself is remembered better than the sample. Perhaps the strongest evidence of outcome expectation comes from findings that training with differential outcomes can promote transfer of responding to untrained stimuli that signal the same outcome (Peterson, 1984).

One mechanism that has been suggested to account for the differential-outcome effect is that outcomes that are 
different from each other actually produce different behaviors (see Alling, Nickel, \& Poling 1991; Brodigan \& Peterson, 1976; Peterson \& Trapold, 1980) and it is the differential behavior that controls comparison choice. Zentall, Sherburne, and Steirn (1992), for example, observed that when training with a differential-outcome procedure, pigeons peck at a higher rate to a stimulus that is associated with a food outcome than to a stimulus that is associated with a no-food outcome. In this case, having pecked or not having pecked at the stimulus could serve as an additional cue for choice.

Although outcomes of food and the absence of food would be most obvious in producing differences in the rate of sample pecking, the same can be said for outcomes that differ in the percentage of trials with and without food outcomes, the magnitude of reinforcement, and the delay of reinforcement. Even when the outcomes differ in the kind of food provided as an outcome (e.g., corn for one correct comparison response, wheat for the other), pigeons often prefer one food over the other, sometimes idiosyncratically (Edwards, Jagielo, Zentall, \& Hogan 1982). In the same manner, pigeons may peck with their beaks closed when the sample signals a water outcome and with their beaks more open when the sample signals a food outcome (Jenkins \& Moore, 1973). In these cases, one could argue that the differential-outcome effect is mediated by differential responding to the sample stimulus. This additional cue would be available for animals trained under the differential-outcome condition, but not for animals trained with nondifferential outcomes.

Zentall and Sherburne (1994) attempted to test this hypothesis by training pigeons with differential outcomes of food and the absence of food, but they included additional trials in which the samples that were associated with no-food outcomes on matching trials were immediately followed by food (without comparison stimuli) on these additional trials. The function of the additional trials was to increase the rate of pecking to samples that were associated with the absence of food for correct choice of the comparison stimulus. The result of these additional trials was that the rate of pecking the two sample stimuli was not significantly different for any of the pigeons. Zentall and Sherburne found that in the absence of differential-sample responding, pigeons trained with differential outcomes still matched with higher accuracy than pigeons in a nondifferential-outcome control group. Furthermore, the levels of accuracy were similar for pigeons in a differentialoutcome group without the additional trials (i.e., ones that exhibited differential responding to the samples). Thus, it appears that differential-sample responding is not necessary to account for the differential-outcome effect.

Alternatively, the arousal or hedonic state elicited by a stimulus that signals an upcoming preferred outcome (e.g., food) may be different from the level produced by a stimulus that signals an upcoming, less preferred outcome (e.g., the absence of food), and the differential levels of arousal could mediate responding. The same can be said for many of the differential outcomes used in this line of research. For example, a sample stimulus that predicts reinforcement with a probability of 1.0 may elicit a greater level of arousal or put the animal in a more positive hedonic state than the level elicited by a stimulus that predicts reinforcement with a probability of .3 , and such a difference in hedonic state may serve as the additional cue for comparison choice. Differential magnitude of reinforcement, delay of reinforcement, and kind of reinforcement may also elicit differential hedonic states, and there is evidence that differential hedonic states are sufficient to control comparison choice (Astley, Peissig, \& Wasserman 2001; Friedrich \& Zentall, 2010). The importance of the distinction between differential hedonic state and differentialoutcome anticipation is that a differential hedonic state may have proprioceptive or emotional stimulus properties at the time of comparison choice, whereas differential outcomes that do not differ in hedonic value would have to involve the representation of a not-yet-present outcome.

Whether differential pecking or differential hedonic states can mediate comparison choice or not, it is of some interest to know whether a differential-outcome effect can be found in the absence of those potential mediators. That is, if differential behavior or a differential hedonic state is not present at the time of comparison choice, it suggests that the anticipation or representation of the outcome, a stimulus that is expected to occur later, can facilitate comparison choice.

Kelly and Grant (2001) examined the effect of differential outcomes that would not be expected to elicit differential hedonic states in pigeons. They used blue and yellow key light illumination for $1.5 \mathrm{~s}$ following correct comparison choices as the manipulated outcomes, followed by nondifferential food reinforcement, and found that the pigeons in the differential-outcome conditions acquired the discrimination significantly more quickly than did those in the nondifferential-outcome group (see also Miller, Friedrich, Narkavic, \& Zentall 2009, with pigeons; Fedorchak \& Bolles, 1986; Friedman \& Carlson, 1973, with rats).

Williams, Butler, and Overmier (1990) also investigated delayed matching with nondifferentially hedonic differential outcomes, but they used similar outcomes presented in two different locations. However, they used a spatial comparison discrimination that was either correlated with the location of the feeder or uncorrelated with the feeder location and found that pigeons for which the comparison location was correlated with the location of the correct comparison stimulus performed more accurately at longer delays than did pigeons for which the comparison location 
was uncorrelated with the location of the correct comparison stimulus. It should be noted, however, that delay testing with a spatial comparison discrimination allows the subject to bridge the delay by approaching the location of the correct choice during the delay. Although this was the same for all of the pigeons, in typical delayed matching, the location of the correct comparison stimulus cannot be predicted prior to its appearance. Furthermore, the fact that both the comparison discrimination and the differential outcomes were based on spatial location may limit the generality of their findings.

In Experiment 1 of the present study, we attempted to confirm the finding that the differential location of food delivery associated with the two kinds of conditional discrimination responses would improve delayed-matching accuracy, relative to nondifferential outcomes. To avoid the potential problem of approaches to the correct key during the delay, we used a more conventional comparison stimulus discrimination: identity matching with red and green samples and red and green comparison stimuli. In Experiment 2, we conducted a more conclusive test of control by differential outcomes by training pigeons on two differential-outcome matching tasks and then testing them for cross-task transfer by presenting them with samples from one task and comparisons from the other. In Experiment 3, we asked whether, when pigeons were trained with differential outcomes, if both comparisons were associated with the same outcome, the sample alone would be able to control comparison choice. Pigeons were trained on two different tasks involving the same differential outcomes, and on test trials, the incorrect comparison was replaced by the comparison from the other task that was associated with the same outcome as the correct comparison. That is, on these test trials, both of the comparisons had been associated with the same outcome. Thus, the identity of the sample was the only basis for comparison choice.

\section{Experiment 1}

Method

\section{Subjects}

Subjects were 8 White Carneaux pigeons (Columba livia), 5-8 years of age, of unknown sex, obtained from the Palmetto Pigeon Plant (Sumter, SC). The pigeons had previously served in a matching-to-sample experiment involving red and green hues and vertical and horizontal lines with nondifferential outcomes. The pigeons were individually housed in a colony room maintained under a 12:12-h light:dark cycle. The animals were maintained at
$85 \%$ of their free-feeding weights for the duration of the experiment, but they had free access to water and grit in the home cage.

\section{Apparatus}

The experiment was conducted in a standard test chamber (BRS, LVE, Laurel, MD) that measured $33 \mathrm{~cm}$ high, $35 \mathrm{~cm}$ from response panel to back wall, and $31 \mathrm{~cm}$ across the response panel. The response panel contained three horizontally aligned response keys $(3.0 \times 2.5 \mathrm{~cm}$, separated by $0.8 \mathrm{~cm}$ ). Each response key could be illuminated by a projector (Industrial Electronics Engineer, Model 10, Van Nuys, CA), mounted behind the key. Access to reinforcement was permitted through two horizontally aligned feeder openings (one under the left response key and the other under the right response key). Reinforcement (Purina Pro Grains) consisted of $1.5-\mathrm{s}$ access to the raised and illuminated grain feeder. A houselight (GE 1820) provided general illumination during intertrial intervals (ITIs). An exhaust fan mounted externally on the chamber masked extraneous sounds. All of the events in the chamber were controlled by a microcomputer located in an adjacent room.

\section{Procedure}

Pretraining Pigeons were first trained to eat from both feeders and then were pretrained to peck at red and green stimuli presented on each of the three keys. The response requirement on the center key was gradually increased to 10 pecks.

Training During training, each trial began with the presentation of either a red or a green sample on the center key. Pigeons were required to peck 10 times to the center key to terminate the sample and were immediately presented with red and green comparison stimuli on the side keys. Red and green comparisons appeared equally often on each side key, and their location was not correlated with sample type. Selecting a red comparison after a red sample and a green comparison after a green sample was reinforced. For 2 pigeons in the differential-outcome (DO) condition, reinforcement was provided at the left feeder on red sample trials and at the right feeder on green sample trials. For 2 other pigeons in the DO condition, reinforcement was provided by the right feeder on red sample trials and by the left feeder on green sample trials. The remaining pigeons were assigned to the nondifferential-outcome (NDO) condition. For these pigeons, reinforcement was provided randomly at the left feeder and at the right feeder on both red and green sample trials.

Training sessions consisted of 96 trials, and each trial was followed by a 10-s ITI, during which the houselight 
was illuminated. Training continued until the subjects reached a criterion of $90 \%$ overall accuracy for two consecutive sessions, followed by five sessions of overtraining.

Testing Each of 20 testing sessions also consisted of 96 trials, but on these sessions, a delay that varied in duration from trial to trial $(0,2,4$, or $8 \mathrm{~s})$ was introduced between the offset of the sample and the onset of the comparison stimuli. During the delay, all the stimuli, including the houselight, remained off. The delays were randomly presented, with the constraint that they occurred equally often within a session (24 trials at each delay).

Results

\section{Training}

Pigeons in the DO condition acquired the discrimination to criterion in a mean of 5.2 sessions, whereas those in the NDO condition reached the criterion in an average of 12.0 sessions. A one-way analysis of variance (ANOVA) indicated that although the difference was large, due to considerable between-subjects variance, it was not statistically reliable, $F(1,6)=2.2, p=.19$.

\section{Testing}

Data from the delay testing indicate that the slope of the retention function was flatter for the DO condition than for the NDO condition (see Fig. 1). A two-way repeated measures ANOVA performed on the delay data pooled over the 20 days of testing indicated that overall performance for the DO condition was superior to that for the NDO condition, $F(1,7)=14.5, p<.01$. There was also a significant effect

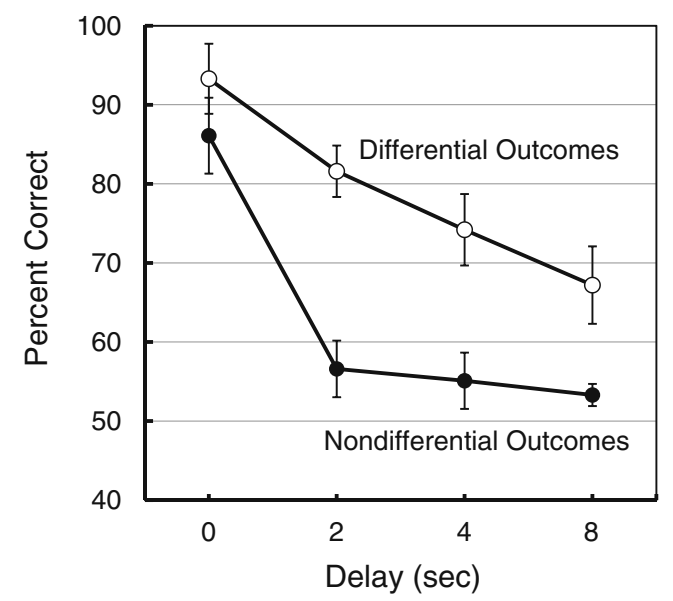

Fig. 1 Experiment 1: Retention functions for pigeons trained with differential outcomes (feeder locations) and nondifferential outcomes of delay, $F(3,18)=44.18, p<.01$, and a significant condition $\times$ delay interaction, $F(3,18)=3.7, p<.05$. As can be seen in Fig. 1, although pigeons in the DO condition still were performing at $67.2 \%$ accuracy on 8 -s delay trials, pigeons in the NDO condition were performing only at $56.6 \%$ accuracy on 2 -s delay trials.

\section{Discussion}

The results of the present experiment confirm the finding by Williams et al. (1990) that when correlated with samples, differential outcomes consisting of comparable reinforcement quality and quantity that are distinguished only by their location can result in higher levels of delayedmatching accuracy, relative to an uncorrelated condition. The present experiment extends the results of Williams et al. to comparison stimuli that are not correlated with feeder location. That is, in the Williams et al. study, it was possible for the pigeons to mediate their comparison response by approaching the location of the correct comparison key during the retention interval. Although it is not clear that such potential mediation would affect the difference in matching accuracy between the differentialoutcome and nondifferential-outcome conditions, given that the differential outcomes consisted of different outcome locations, in the present experiment we more clearly separated the effect of differential-outcome location from the effect of differential sample location as a choice cue.

The present results, together with those of Williams et al. (1990) and Kelly and Grant (2001), indicate that the anticipation of differential outcomes that do not differ in their presumed hedonic value can provide cues for comparison choice. Such results suggest that the differentialoutcome effect does not depend on differential hedonic states elicited by the samples that can then be used as cues for comparison choice. They suggest further that pigeons are able to use some representation of the expected outcome to aid in making their choice of comparison.

\section{Experiment 2}

Although facilitated memory, as indicated by significantly flatter retention functions, has been accepted as evidence of a differential-outcome effect, more direct evidence of control by outcome expectancy would be shown by the transfer of training in which outcome anticipation is the only cue to comparison choice (see Peterson, 1984). According to Urcuioli (2005),

Perhaps the clearest and most convincing evidence for discriminative control by outcomes expectancies is the finding that other stimuli will immediately control 
baseline performances, despite no reinforced history of doing so, if they signal the same differential outcomes as the training stimuli. (p. 8)

A typical transfer-of-control experiment involves three phases (Trapold \& Overmier, 1972). In the first phase, two responses (R1 and R2) are each reinforced by a different outcome (O1 and $\mathrm{O} 2)$ following the presentation of one of two discriminative stimuli (S1 or S2). In a second phase, two new stimuli (S3 and S4) are paired with the two outcomes from the first phase (S3 is paired with O1, and S4 is paired with $\mathrm{O} 2$ ). Finally, in a testing phase, one assesses the preference for R1 and R2 in the presence of S3 and S4.

Using such a procedure, Peterson (1984) demonstrated that in the absence of any other cue, outcome expectancies mediated choice responding. Using food and no food as the two outcomes, Peterson trained pigeons on delayed matching in which choices to vertical comparisons after red samples produced a food outcome and choices to horizontal comparisons after green sample presentations produced a no-food outcome. He also trained these subjects in a simple delayed successive discrimination in which the pigeons received a food outcome after being presented with a circle and a no-food outcome after being presented with a triangle. In a transfer test, red and green samples were replaced by circles and triangles. He found that the pigeons chose the comparison that, in training, was associated with the same outcome as the line orientation about $90 \%$ of the time. Similar results were reported by Urcuioli and Zentall (1992).

In a variation of this procedure, Edwards et al. (1982) trained pigeons on two matching tasks with common differential outcomes, corn and wheat, in both tasks. When the samples from one task were substituted for those of the second task, they found that the pigeons were significantly more likely to choose comparisons associated with the same outcome as the sample than were pigeons trained with nondifferential outcomes.

Although one might expect that the two different grains would be similarly motivating, Edwards et al. (1982) observed that pigeons made significantly more correct matching responses to stimuli associated with the wheat reinforcement than to stimuli associated with corn. This bias to choose comparisons associated with one of the outcomes suggests that the birds may have had a preference for that outcome. In this case, the anticipation of the preferred outcome may have led to differential responding to the samples (not analyzed by the authors) and, perhaps, to a more positive hedonic state than when the less preferred outcome was anticipated.

In a second experiment, the authors tried to avoid this possible mediation by differential sample pecking or by differential hedonic states by using two outcomes for which the pigeons did not show a systematic preference: wheat and peas. Although they no longer found a consistent comparison bias and they replicated the transfer effect, it is possible that individual idiosyncratic food preferences remained.

The present experiment was designed to determine whether expectancy alone could serve as a basis for comparison choice, using two distinctive but qualitatively and quantitatively similar outcomes. As in Experiment 1, pigeons had access to food in two different feeder locations, but in Experiment 2, the pigeons were trained on two different matching tasks, hue identity and line orientation identity, using the same differential-outcome locations. During testing, samples from one matching task were replaced by samples from the other task. If outcome expectancies were to develop, they should be the only cues available for comparison choice. During testing, for pigeons in the consistent group, choice of the comparison that, in training, was associated with the same reinforcement location as the sample was reinforced. For pigeons in the inconsistent group, choice of the comparison that, in training, was associated with the reinforcement location different from the sample was reinforced.

If anticipation of an outcome can serve as a discriminative stimulus, pigeons in both groups should use their expectancies to guide their comparison choices, and those in the consistent group should perform above chance, whereas those in the inconsistent group should perform below chance.

\section{Method}

\section{Subjects}

Subjects were eight White Carneaux pigeons similar to those used in Experiment 1. The pigeons were housed as were the pigeons in Experiment 1.

\section{Apparatus}

The apparatus was the same as that used in Experiment 1 . Vertical and horizontal line stimuli were added to each of the projectors.

\section{Procedure}

Pretraining Pigeons were trained to peck at red, green, and vertical and horizontal line stimuli projected on each the three response keys individually. The pecking requirement was progressively increased to 10 pecks on the center key.

Training Pigeons were initially trained on identity matching with vertical and horizontal samples. For half of the subjects, correct vertical choices were reinforced with food delivered by the left feeder, and correct horizontal choices were reinforced with food delivered by the right feeder. For 
the remaining birds, food was available from the right feeder for correct responses on vertical sample trials and from the left feeder on correct horizontal sample trials. Each training sessions consisted of 96 trials, 48 of each trial type randomly presented, with the constraint that no more than three of each trial type should occur on successive trials. After each pigeon reached a criterion of $90 \%$ accuracy on both vertical and horizontal line trials for 2 consecutive sessions, red and green identity matching-tosample trials were introduced. Correct choices on trials involving one of the hue samples (e.g., red) were reinforced with access to food at the left feeder, whereas correct choices on trials involving the other hue sample (i.e., green) were reinforced with access to food in the right feeder. These training sessions also consisted of 96 trials with equal numbers of vertical, horizontal, red, and green sample trials. A schematic representation of training trials is presented in Table 1. Training ended when matching accuracy reached $85 \%$ or better on each trial type for 2 consecutive sessions or after 30 sessions with all four samples.

Testing The pigeons were randomly assigned to two groups, the consistent group $(n=4)$ and the inconsistent group $(n=4)$. On test trials, red and green samples were replaced by vertical and horizontal line samples, and vertical and horizontal line stimuli were replaced by red and green samples. For pigeons in the consistent group, responses to the comparison that in training had been associated with the same feeder as the replaced sample were reinforced (e.g., selecting a vertical comparison on a red sample trial when reinforcement had been obtained from the left feeder on vertical and red sample trials during training). For pigeons in the inconsistent group, responses to the comparison that, in training, had been associated with

Table 1 Training and testing trials in Experiment 2

\begin{tabular}{llll}
\hline & Sample & Comparison & Feeder \\
\hline Training & $\mathrm{V}$ & $\mathrm{V}+\mathrm{H} \cdot$ & left \\
& $\mathrm{H}$ & $\mathrm{H}+\mathrm{V} \cdot$ & right \\
& $\mathrm{R}$ & $\mathrm{R}+\mathrm{G} \cdot$ & left \\
& $\mathrm{G}$ & $\mathrm{G}+\mathrm{R} \cdot$ & right \\
Testing consistent & $\mathrm{R}$ & $\mathrm{V}+\mathrm{H} \cdot$ & left \\
& $\mathrm{G}$ & $\mathrm{H}+\mathrm{V} \cdot$ & right \\
& $\mathrm{V}$ & $\mathrm{R}+\mathrm{G} \cdot$ & left \\
& $\mathrm{H}$ & $\mathrm{G}+\mathrm{R} \cdot$ & right \\
Testing inconsistent & $\mathrm{G}$ & $\mathrm{V}+\mathrm{H}$ & left \\
& $\mathrm{R}$ & $\mathrm{H}+\mathrm{V} \cdot$ & right \\
& $\mathrm{H}$ & $\mathrm{R}+\mathrm{G} \cdot$ & left \\
& $\mathrm{V}$ & $\mathrm{G}+\mathrm{R} \cdot$ & right \\
\hline
\end{tabular}

$\mathrm{R}=$ red, $\mathrm{G}=$ green, $\mathrm{V}=$ vertical, $\mathrm{H}=$ horizontal. Counterbalancing of feeder location not shown

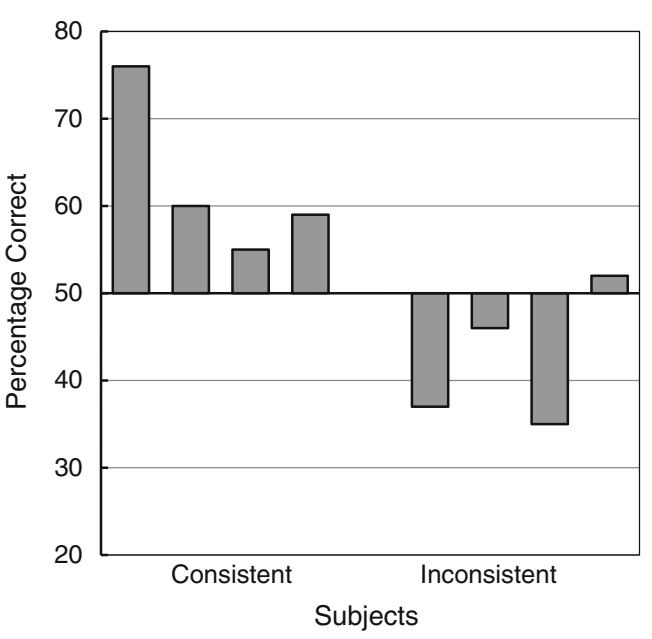

Fig. 2 Experiment 2: Transfer of training. Pigeons in the consistent group were reinforced for choosing the comparison stimulus that, in training, had been associated with the same outcome as the sample. Pigeons in the inconsistent group were reinforced for choosing the comparison stimulus that, in training, had been associated with an outcome that was different from the outcome of the sample

a feeder different from the replaced sample were reinforced (e.g., selecting a horizontal comparison on a red sample trial when reinforcement had been obtained from the left feeder on vertical and red sample trials during training). Each testing session consisted of 96 trials with the same number of red, green, horizontal line, and vertical line sample trials. Testing ended when subjects performed with at least $85 \%$ accuracy on each trial type for 2 consecutive sessions or after 50 testing sessions.

\section{Results}

Matching accuracy on the last training session prior to testing was similar for pigeons in the consistent (96.1\%) and inconsistent $(95.1 \%)$ groups, $t(6)=0.62, p>.05$. Matching accuracy on the first testing session was higher for pigeons in the consistent group (62.8\%) than for pigeons in the inconsistent group (42.7\%). Individual performance on the first test session can be seen in Fig. 2. A $t$ test performed on the data from the first test session indicated that the difference was statistically significant, $t(6)=3.36, p<.05$. On the first testing session, 3 of the 4 pigeons in the consistent group performed significantly above chance (binomial test, $p<.05$ ), and 2 of the 4 pigeons in the inconsistent group performed significantly below chance. Pigeons in the consistent condition also acquired the new task significantly faster (17.8 sessions) than pigeons in the inconsistent group (41.0 sessions), $t(6)=2.46, p<.05$.

Mean peck rates to the hue samples associated with the left and right feeders were 2.04 and 2.55 pecks/s, 
respectively. On line sample trials, pigeons pecked at a rate of 2.75 and 3.18 pecks/s to the samples associated with the left and right feeders, respectively. A one-way repeated measures ANOVA performed on the peck rates revealed that the differences in peck rates to the four samples on the last session of training were not significantly different, $F(3,21)=1.89, p>.05$.

\section{Discussion}

In Experiment 2, when samples from one matching task were replaced by the samples from the other matching task, pigeons tended to choose the comparison that was associated with the same outcome as the sample stimulus. Thus, matching accuracy was higher when those choices were reinforced (consistent group) than when the alternative choices were reinforced (inconsistent group).

This finding is consistent with results from previous research that used differential outcomes that were hedonically differential (e.g., differential probability of reinforcement or differential magnitude of reinforcement), but importantly, the results of the present experiment indicate that it is not necessary for the sample stimuli to induce differential hedonic states for transfer-of-training effects to be found. Furthermore, this experiment provides the first evidence that nondifferentially hedonic differential outcomes can result in transfer of training to new combinations of sample and comparison stimuli.

Honig, Matheson, and Dodd (1984) suggested that differential outcomes may facilitate acquisition and delayedmatching accuracy by enhancing sample discriminability. This hypothesis was based on the observation that rats learned a simple discrimination between two runaways when the end boxes had different colors and shapes on correct and incorrect choice trials (Lawrence \& Hommel, 1961). The results of Experiment 2, as well as other transfer-of-training experiments with differential outcomes, demonstrate that the differential-outcome procedure does more than increase sample discriminability. They suggest that outcome anticipation alone is sufficient to influence comparison choice.

Transfer-of-training results provide the strongest evidence that it is the common outcomes themselves that mediate the associations between samples and comparisons that have never been presented before on the same trial. The results suggest that some representation of the outcome elicited by the sample can be used as a cue for comparison choice.

\section{Experiment 3}

Honig and Dodd (1986) suggested that outcome expectancies generated by the sample stimulus are sufficiently strong that they may overshadow or even supplant the sample stimulus itself. In other words, when the differential-outcome procedure is used, direct control by the sample stimulus may be replaced by outcome expectancies.

Alternatively, expectancies generated by sampleoutcome associations may simply serve as an additional discriminative stimulus. If so, subjects benefit from the differential-outcome procedure because it provides them with two cues for comparison choice, instead of one.

To distinguish between these hypotheses, Urcuioli (1990) trained pigeons with differential outcomes involving different probabilities of reinforcement (1.0 and .2) and compared their performance with that of a nondifferentialoutcome group. After observing faster acquisition and superior delayed-matching accuracy for pigeons trained with differential outcomes, he attempted to remove the outcome expectancies to determine whether sample-comparison associations were still effective in making comparison choices. To eliminate the sample-outcome expectancies, he presented the samples from original training followed immediately with nondifferential outcomes (i.e., there were no comparisons, and the probability of reinforcement was .6 following both samples). He reasoned that if outcome expectancies were overshadowing the samples, pigeons that received nondifferential-outcome training following differential-outcome matching training should perform poorly when they were returned to the original matching task (now without differential reinforcement). If, on the other hand, the pigeons were using their outcome expectancies only as an additional cue for comparison choice, the differential- and nondifferential-outcome groups should perform similarly when returned to the matching task.

The results obtained were somewhat inconsistent. Pigeons trained with differential probabilities of reinforcement of 1.0 and .2 showed little evidence of overshadowing (matching accuracy did not differ significantly from that of pigeons in the nondifferential-outcome control group), whereas pigeons trained with differential probabilities of reinforcement of 1.0 and 0.0 showed poorer matching accuracy than did their nondifferential-outcome controls.

The purpose of Experiment 3 in the present study was to determine whether the feeder location differential outcomes used in the present research would replace memory for the sample, such that pigeons would rely solely on outcome expectation as a basis for comparison choice. To accomplish this, we used a testing procedure that was somewhat different from that used by Urcuioli (1990). In Experiment 3 , pigeons were trained on two independent matching tasks (color and line orientation identity, as in Experiment 2), both with differential feeder location outcomes. On critical test trials, the incorrect comparison was replaced by a comparison from the other matching task that, in training, had been followed by reinforcement in the same location as the correct comparison. Thus, although the correct compar- 
ison was still available, outcome expectancies could not be used effectively for comparison choice.

If pigeons trained with differential outcomes used outcome expectancy alone as a basis for comparison choice, then on these critical test trials, similar outcome expectancies would be elicited by both comparison stimuli, and matching accuracy should suffer. If, however, the anticipation of a given outcome served as an additional cue to comparison choice, pigeons should be able to recognize the comparison stimulus appropriate to the sample presented.

\section{Method}

\section{Subjects}

Eight White Carneaux pigeons similar to those used in Experiments 1 and 2 were used in Experiment 3. The pigeons were maintained as were the pigeons in Experiments 1 and 2.

\section{Apparatus}

The experiment was conducted in the same apparatus as that in Experiments 1 and 2.

\section{Procedure}

Pretraining The pigeons were initially trained to peck at red, green, vertical line, and horizontal line stimuli presented on the center and side keys. Peck requirements were then gradually increased to 10 pecks on the center key. During pretraining, the birds were reinforced by random presentations of food in the two feeders.

Training The pigeons were trained on identity matching to sample with line samples and line comparisons and on identity matching to sample with hue samples and hue comparisons. Ten pecks to either a vertical or a horizontal line presented on the center key terminated its presentation, which was followed by presentations of vertical and horizontal line stimuli on the side keys. Choice of the comparison that had the same line orientation as the sample led to reinforcement. For half of the subjects, correct vertical choices were followed by reinforcement delivered at the left feeder, and correct horizontal choices were followed by reinforcement delivered at the right feeder. For the other half of the pigeons, the stimulus-outcome associations were reversed. The second matching task involved red and green hues as samples and comparison stimuli. Again, 10 pecks to the sample terminated its presentation, which was followed by presentation of the red and green comparison stimuli. For half of the pigeons, choice of the red comparison on red sample trials was followed by reinforcement at the left feeder, and choice of the green comparison on green sample trials was followed by reinforcement at the right feeder. For the remaining pigeons, the feeders associated with correct choice on red and green sample trials were reversed. On all trials, a 10-s ITI followed the comparison choice. Incorrect comparison choices led to the ITI and to the repetition of the trial. Subjects received 96 trials per session, 24 trials with each sample, for a total of 50 sessions.

Testing The pigeons received four different kinds of test trials. Baseline trials involved the four trial types experienced during training. Transfer trials involved replacement of the sample from one matching task by a sample from the other task (a hue replaced a line, or a line replaced a hue). Matching accuracy on these trials served as a test of control by outcome anticipation (similar to testing in Experiment 2). Retrospective code trials involved replacement of the incorrect comparison stimulus by the comparison stimulus from the other matching task that had been associated with the same outcome in training as the correct comparison stimulus. On these trials, outcome anticipation was presumably the same for both comparisons, so the only cue for comparison choice should have been the identity of the sample. Control trials involved replacement of the incorrect comparison stimulus by the comparison stimulus from the other matching task that had been associated with an outcome different from the correct comparison stimulus. These trials served as a control for the possible novelty effect of presentation of a comparison stimulus from the other task. Comparison of matching accuracy on control trials with matching accuracy on retrospective code trials should indicate the contribution of outcome anticipation to the identity of the sample, whereas comparison of matching accuracy on control trials with matching accuracy on baseline trials should indicate the effect of the novel incorrect comparison on matching accuracy. A schematic representation of training and testing trials appears in Table 2.

Comparison choice on baseline trials was reinforced as during training. Choices on all testing trials were reinforced nondifferentially, and reinforcement location was consistent with the comparison stimulus associations acquired during training. There were four 96-trial testing sessions, each consisting of 24 trials of each of the four kinds of trials described above.

\section{Results}

\section{Training}

Pigeons' matching accuracy, pooled over the last four sessions of training on line orientation matching, was $92.9 \%$ correct, and on hue matching, it was $98.2 \%$ correct. 
Table 2 Training and testing trials in Experiment 3

\begin{tabular}{|c|c|c|c|c|}
\hline \multicolumn{3}{|c|}{ Baseline Training } & \multicolumn{2}{|l|}{ Transfer } \\
\hline Phase 1 & Phase 2 & Transfer & Retrospective Code & Control \\
\hline $\mathrm{V}-\mathrm{V}-\mathrm{LF}$ & $\mathrm{V}-\mathrm{V}-\mathrm{LF}$ & $\mathrm{R}-\mathrm{V} / \mathrm{H}-\mathrm{LF}$ & $\mathrm{R}-\mathrm{R} / \mathrm{V}-\mathrm{LF}$ & $\mathrm{R}-\mathrm{R} / \mathrm{H}-\mathrm{LF}$ \\
\hline \multirow[t]{3}{*}{$\mathrm{H}-\mathrm{H}-\mathrm{RF}$} & $\mathrm{H}-\mathrm{H}-\mathrm{RF}$ & $\mathrm{G}-\mathrm{H} / \mathrm{V}-\mathrm{RF}$ & $\mathrm{G}-\mathrm{G} / \mathrm{H}-\mathrm{RF}$ & $\mathrm{G}-\mathrm{G} / \mathrm{V}-\mathrm{RF}$ \\
\hline & $\mathrm{R}-\mathrm{R}-\mathrm{LF}$ & $\mathrm{V}-\mathrm{R} / \mathrm{G}-\mathrm{LF}$ & $\mathrm{V}-\mathrm{V} / \mathrm{R}-\mathrm{LF}$ & $\mathrm{V}-\mathrm{V} / \mathrm{G}-\mathrm{LF}$ \\
\hline & $\mathrm{G}-\mathrm{G}-\mathrm{RF}$ & $\mathrm{H}-\mathrm{G} / \mathrm{R}-\mathrm{RF}$ & $\mathrm{H}-\mathrm{H} / \mathrm{G}-\mathrm{RF}$ & $\mathrm{H}-\mathrm{H} / \mathrm{R}-\mathrm{RF}$ \\
\hline
\end{tabular}

$\mathrm{V}=$ vertical lines, $\mathrm{H}=$ horizontal lines, $\mathrm{R}=\mathrm{red}, \mathrm{G}=$ green, $\mathrm{LF}=$ left feeder, $\mathrm{RF}=$ right feeder. First letter $=$ sample, second letter $=$ correct comparison, and third letter $=$ reinforcement location. Incorrect comparison stimuli have been omitted from Phases 1 and 2 . On test trials, comparison choice was reinforced nondifferentially. Counterbalancing conditions have been omitted. Transfer trials involved samples from one training task and comparisons from the other training task. Retrospective code trials involved the correct comparison from training, as well as the comparison from the other training task that was associated with the same outcome in training. Control trials involved replacing the incorrect comparison with the comparison from the other training task associated with the same outcome as the incorrect comparison (this condition served as a control for the novelty of the incorrect comparison)

\section{Testing}

A repeated measures ANOVA performed on the data from the testing sessions indicated that there were significant differences among the testing conditions, $F(3,18)=26.07$, $p<.001$. Matching accuracy on baseline trials was $84.8 \%$ correct during the four testing sessions. Matching accuracy on transfer trials was $60.0 \%$, a level that was significantly above chance, $t(7)=2.89, p<.05$. But there was also a significant difference in matching accuracy between baseline trials and transfer trials, $t(7)=8.96, p<.01$. Matching accuracy on retrospective code trials was $59.8 \%$ correct, also significantly above chance, $t(7)=4.05, p<.01$. Furthermore, the difference between baseline trial matching accuracy $(84.8 \%)$ and matching accuracy on retrospective code trials $(59.8 \%)$ was also significant, $t(7)=10.19, p<.01$. On control trials, pigeons matched correctly $74.0 \%$ of the time, significantly different from chance, $t(7)=6.59, p<.01$, and significantly different from baseline trials, $t(7)=4.63$, $p<.01$. Matching accuracy on the four test trial types can be seen in Fig. 3.

\section{Discussion}

The results from Experiment 3 replicated the finding from Experiment 2 that in the absence of the trained samplecomparison association, pigeons can use outcome expectancy as a cue for comparison choice. However, when differential-outcome expectancy was not available and the sample was the only cue that was available for comparison choice (retrospective code), pigeons showed a significant tendency to choose the correct comparison. That is, outcome expectancies do not replace sample memory as the basis for comparison choice.

Urcuioli (1990) found that under certain conditions, differential outcomes did come to replace the sample as the basis for comparison choice. When the differential out- comes were the presence versus the absence of food (i.e., 1.0 probability of food vs. 0.0 probability of food), eliminating the differential-outcome expectation resulted in poorer matching accuracy for pigeons trained with differential outcomes than for pigeons trained with nondifferential outcomes. However, when the outcomes were less differential (1.0 vs. .2), sample stimuli continued to control comparison choice. That is, pigeons continued to choose comparisons in the absence of differential-outcome expectations. Thus, it appears that the salience of the difference in the outcomes may be a factor in the degree of control found for the samples. That conclusion is consistent with the results of Experiment 3, because it would be expected that differential feeder locations would be a less salient difference than the presence versus the absence of

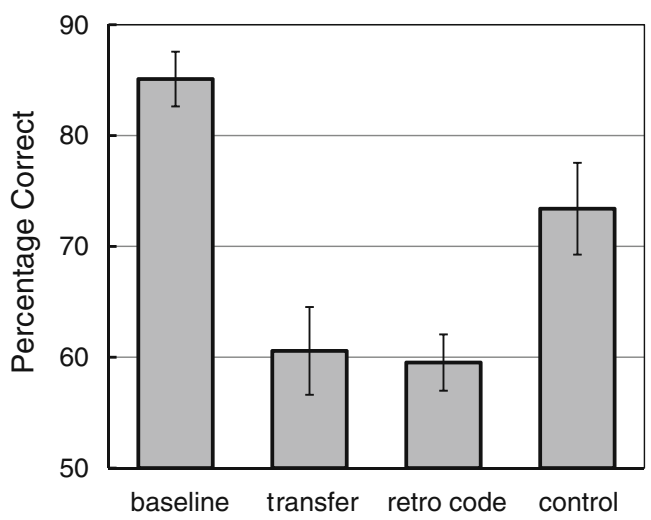

Fig. 3 Experiment 3, involving four kinds of trials: Baseline trials that were the same as training trials; transfer trials that assessed the prospective code involving samples from one training task and comparisons from the other; retrospective code trials (retro code) that involved the correct comparison from training and the other comparison that, in training, had been associated with the same outcome; and control trials that involved replacing the incorrect comparison with the comparison from the other task associated with the same outcome as the incorrect comparison (this condition served as a control for the novelty of the incorrect comparison) 
food, and in the present experiment, samples continued to control comparison choice in the absence of a differentialoutcome cue. In fact, given the decrement in matching accuracy found on control trials, the control of comparison choice by the sample stimulus is likely to have been underestimated. That is, it is likely that some of the decrement in matching accuracy could be attributed to the novelty of a new incorrect comparison stimulus.

\section{General discussion}

The purpose of the present experiments was to clarify the mechanisms responsible for the faster acquisition, more accurate delay performance, and transfer effects often found when differential outcomes follow correct comparison choice on the two sample types in matching to sample. Most research with differential outcomes has used outcomes that differ in their hedonic value (e.g., different probabilities, magnitudes, or qualities of reinforcement). Some research has suggested that outcomes unlikely to have differential hedonic value, such as different colored lights (Kelly \& Grant, 2001) or a light following a correct choice on one kind of trial and a tone following a correct choice on the other kind of trial (Miller et al., 2009), can also produce a differential-outcome effect. The present research examined feeder location as a differential outcome, using a more typical comparison choice procedure that avoided the correlation between correct comparison location and outcome location used by Williams et al. (1990).

In Experiment 1, we found that training with differentialoutcome locations facilitated delayed-matching accuracy. In Experiment 2, using a transfer-of-training design, we found direct evidence for the use of outcome anticipations as the basis for comparison choice. When, after pigeons had been trained on two matching tasks (one with hues as samples and comparisons and the other with lines as samples and comparisons), the samples from the two tasks were interchanged; positive transfer was found for pigeons in the consistent group, whereas negative transfer was found for pigeons in the inconsistent group. This finding indicates that in the absence of other cues for correct comparison choice, the correlation between sample outcome expectation and comparison outcome expectation can serve as an effective cue for comparison choice. Furthermore, the use of nonhedonically different differential outcomes suggests that the outcome expectations do not need to evoke differential hedonic states, which themselves may serve as cues for comparison choice. This finding implies that an outcome expectancy can consist of a rather arbitrary event, such as a color, a tone, or a location in the operant chamber.
In Experiment 3, we asked whether outcome expectancy replaces sample memory by removing outcome expectancy as a differential cue for comparison choice and found that although matching accuracy was substantially reduced, there was residual control by the sample. It may be that when the differential-outcome procedure is used, the degree of control of comparison choice by sample stimuli and by outcomes depends on the relative salience of the two sources of cues for comparison choice.

In the present research, it was assumed that the pigeons had no significant preference for either feeder, and in fact, no systematic group or individual bias was noted. Specifically, the pigeons showed virtually no differences in the acquisition of the trial types associated with each of the two feeders, as one often sees with differential outcomes that clearly differ in hedonic value (e.g., low vs. high probability of reinforcement; Urcuioli, 1990). Nonetheless, there may have been individual differences in the pigeons' preference between the two feeders. If so, however, it is unlikely that such potential differential preferences could have provided sufficient differentially hedonic cues to account for the large effects found in the present research.

The present research (and earlier work) extends the conditions under which one can see evidence of facilitation of matching accuracy by outcome anticipation in which the outcomes are hedonically nondifferential. In a typical Pavlovian conditioning experiment, outcome expectations can be shown in the development of a conditioned response to a conditioned stimulus. Furthermore, sensory-preconditioning research suggests that animals can also represent arbitrary events (Lavin, 1976; Rescorla \& Durlach, 1981) and can even represent their temporal order (Matzel, Held, \& Miller 1988). The present research extends this research to include instrumental behavior - that is, to cues that can be used as the basis for choice in conditional discriminations.

\section{References}

Alling, K., Nickel, M., \& Poling, A. (1991). The effects of differential and nondifferential outcomes on response rates and accuracy under a delayed-matching-to-sample procedure. Psychological Record, 41, 537-549.

Astley, S. L., Peissig, J. J., \& Wasserman, E. A. (2001). Superordinate categorization via learned stimulus equivalence: Quantity of reinforcement, hedonic value, and the nature of the mediator. Journal of Experimental Psychology: Animal Behavior Processes, 27, 252-268.

Brodigan, D. L., \& Peterson, G. B. (1976). Two-choice conditional discrimination performance of pigeons as a function of reward expectancy, prechoice delay, and domesticity. Animal Learning \& Behavior, 4, 121-124.

Edwards, C. A., Jagielo, J. A., Zentall, R., \& Hogan, D. E. (1982). Acquired equivalence and distinctiveness in matching to sample by pigeons: Mediation by reinforcer-specific expectancies. 
Journal of Experimental Psychology: Animal Behavior Processes, $8,244-259$.

Fedorchak, P. M., \& Bolles, R. C. (1986). Differential outcome effect using a biologically neutral outcome difference. Journal of Experimental Psychology: Animal Behavior Processes, 12, 125-130.

Friedman, G. J., \& Carlson, J. G. (1973). Effects of stimulus correlated with positive reinforcement upon discrimination learning. Journal of Experimental Psychology, 97, 281-286.

Friedrich, A. M., \& Zentall, T. R. (2010). A relational differentialoutcomes effect: pigeons can classify outcomes as "good" and "better". Animal Cognition, 13, 359-365.

Honig, W. K., \& Dodd, P. W. D. (1986). Anticipation and intention in working memory. In D. F. Kendrick, M. E. Rilling, \& M. R. Denny (Eds.), Theories of animal memory (pp. 77-100). Hillsdale, NJ: Erlbaum.

Honig, W. K., Matheson, W. R., \& Dodd, P. W. D. (1984). Outcome expectancies as mediators for discriminative responding. Canadian Journal of Psychology, 38, 196-217.

Jenkins, H. M., \& Moore, B. R. (1973). The form of the auto-shaped response with food or water reinforcers. Journal of the Experimental Analysis of Behavior, 20, 163-181.

Kelly, R., \& Grant, D. S. (2001). A differential outcomes effect using biologically neutral outcomes in delayed matching-to-sample with pigeons. The Quarterly Journal of Experimental Psychology, $54 B, 69-79$.

Lavin, M. J. (1976). The establishment of flavor-flavor associations using a sensory preconditioning training procedure. Learning and Motivation, 7, 173-183.

Lawrence, D. H., \& Hommel, L. (1961). The influence of differential goal boxes on discrimination learning involving delay of reinforcement. Journal of Comparative and Physiological Psychology, 51, 552-555.

Matzel, L. D., Held, F. P., \& Miller, R. R. (1988). Information and expression of simultaneous and backward associations: implications for contiguity theory. Learning and Motivation, 19, 317344.

Miller, H. C., Friedrich, A. M., Narkavic, R. J., \& Zentall, T. R. (2009). A differential outcomes effect using hedonicallynondifferential outcomes with delayed matching-to-sample by pigeons. Learning \& Behavior, 37, 161-166.

Peterson, G. B. (1984). How expectancies guide behavior. In H. L. Roitblat, T. G. Bever, \& H. S. Terrace (Eds.), Animal cognition (pp. 135-148). Hillsdale, NJ: Erlbaum.
Peterson, G. B., \& Trapold, M. A. (1980). Effects of altering outcome expectancies on pigeons' delayed conditional discrimination performance. Learning and Motivation, 11, 267-288.

Rescorla, R. A., \& Durlach, P. J. (1981). Within-event learning in Pavlovian conditioning. In N. E. Spear \& R. R. Miller (Eds.), Information processing in animals: Memory mechanisms (pp. 81-112). Hillsdale, NJ: Erlbaum.

Trapold, M. A. (1970). Are expectancies based upon different positive reinforcing events discriminately different? Learning and Motivation, 1, 129-140.

Trapold, M. A., \& Overmier, J. B. (1972). The second learning process in instrumental learning. In A. A. Black \& W. F. Prokasy (Eds.), Classical conditioning, current research and theory (Vol. 2, pp. 427-452). New York: Appleton-Century-Crofts.

Urcuioli, P. J. (1990). Some relationships between outcome expectancies and sample stimuli in pigeons' delayed matching. Animal Learning \& Behavior, 18, 302-314.

Urcuioli, P. J. (2005). Behavioral and associative effects of differential outcomes in discrimination learning. Learning \& Behavior, 33, $1-21$.

Urcuioli, P. J., \& Zentall, T. R. (1992). Transfer across delayed discriminations: evidence regarding the nature of prospective working memory. Journal of Experimental Psychology: Animal Behavior Processes, 18, 154-173.

Williams, D. A., Butler, M. M., \& Overmier, J. B. (1990). Expectancies of reinforcement location and quality as cues for a conditional discrimination in pigeons. Journal of Experimental Psychology: Animal Behavior Processes, 16, 3-13.

Zentall, T. R., \& Sherburne, L. M. (1994). Role of differential sample responding in the differential outcomes effect involving delayed matching by pigeons. Journal of Experimental Psychology: Animal Behavior Processes, 20, 390-401.

Zentall, T. R., Sherburne, L. M., \& Steirn, J. N. (1992). Development of excitatory backward associations during the establishment of forward associations in delayed conditional discrimination by pigeons. Animal Learning \& Behavior, 20, 199-206.

This research was supported by National Institute of Mental Health Grant 63726 and by National Institute of Child Health and Development Grant 60996. 